\title{
A painful tic convulsif due to double neurovascular impingement
}

\author{
G. Giglia $\cdot$ M. Romano $\cdot$ P. Paladino $\cdot$ \\ V. Virzì · F. Narese $\cdot$ A. Palermo $\cdot$ B. Fierro $\cdot$ \\ F. Brighina
}

Received: 7 June 2011/Accepted: 19 July 2011/Published online: 4 August 2011

(c) The Author(s) 2011. This article is published with open access at Springerlink.com

\begin{abstract}
Here we present the case of a 50-year-old man suffering from "painful tic convulsif", on the left side of the face, i.e., left trigeminal neuralgia associated with ipsilateral hemifacial spasm. An angio-MRI scan showed a neurovascular confliction of left superior cerebellar artery with the ipsilateral $\mathrm{V}$ cranial nerve and of the left inferior cerebellar artery with the ipsilateral VII cranial nerve. Neurophysiological evaluation through esteroceptive blink reflex showed the involvement of left facial nerve. An initial carbamazepine treatment ( $800 \mathrm{mg} /$ daily) was completely ineffective, so the patient was shifted to lamotrigine 50 b.i.d. that was able to reduce attacks from 4 to 6 times per day to 1 to 2 per week. Considering the good response to the drug, the neurosurgeon decided to delay surgical treatment.
\end{abstract}

Keywords Painful tic convulsif - Trigeminal neuralgia . Hemifacial spasm · Double vascular impingement

G. Giglia $\cdot$ P. Paladino $\cdot$ A. Palermo .

B. Fierro · F. Brighina $(\square)$

Department of Experimental Biomedicine and Clinical

Neurosciences, University of Palermo, Via G. La Loggia, 1,

90129 Palermo, Italy

e-mail: fbrighina@unipa.it

M. Romano

Department of Neurology, Hospital "Villa Sofia",

Palermo, Italy

V. Virzì · F. Narese

Radiology Unit, Casa di Cura "Regina Pacis",

San Cataldo, CL, Italy

\section{Introduction}

"Painful tic convulsif" is a rare condition characterized by paroxysmal irritative dysfunction of the V and VII ipsilateral cranial nerves [1]. Since the first description in 1920 by Cushing [2] only few cases were reported in literature. Most of them were caused by masses or by a singular vascular loop in the posterior fossa, involving both the facial and trigeminal nerves [3]. Even if this condition is considered rare, we think that a considerable number of cases might escape notice unless careful history, neuroimaging and neurophysiological studies are performed.

\section{Case report}

We report on a 50-year-old Italian man. His chief complaints were episodes of pain in infraorbital region and muscular twitches of ipsilateral hemiface. He had no family history for headache or similar pain and there was nothing remarkable in his past medical history.

\section{Current medical history}

The patient presented to our headache center with a 2-year history of pain in the left infraorbital region and subsequent ipsilateral facial spasm. At onset patient experienced short episodes of excruciating pain in the left infraorbital region. Three months later an ipsilateral facial spasm took place. Patient referred to his general practitioner and underwent a brain MRI scan, that was normal, then a diagnosis of "trigeminal neuralgia" was made and the patient started a trial with carbamazepine 
(CBZ) (800 mg/daily) that was ineffective. Pain episodes were described as "always the same, very intense, sometimes unbearable, localized exactly under my left eye", lasting about $30 \mathrm{~s}$ and presenting four to six times per day. No trigger areas were reported. The facial twitch started about 3 months after the beginning of pain and was described as "involuntary grimace unrelated to pain”.

\section{Current illness}

Physical examination was normal; blood pressure: 120/70 $\mathrm{mmHg}$ and heart rate: $80 \mathrm{bpm}$. Also routine laboratory data were normal. Neurological examination revealed a mild peripheral palsy of the VII nerve with very frequent hemifacial spasms in the left side. No other neurological abnormalities were present.

\section{Neuroimaging}

\section{Angio-MRI scan}

A 1.5-T MRI scan was performed with FSE sequences, SE and FLAIR, T1 and T2-weighted. TOF3D sequences were also collected to study the vascular tree. No abnormalities of brain parenchyma, parenchymal cerebellar or brainstem were found. The MR angiographic examination and the thin layer study of the cranial base showed: close contiguity of the trigeminal nerve with left medial ipsilateral posterior cerebellar artery and inferiorly with a small venous vessel (Fig. 1); close contiguity of the left VII and VIII cranial nerve with ipsilateral posterior inferior cerebellar artery (Fig. 2).
Neurophysiology

A blink reflex examination, performed stimulating the supraorbital branch of trigeminal nerve and recording from orbicular eye muscles, showed delayed ipsilateral R1 and $\mathrm{R} 2$ responses with normal contralateral R2 stimulating the left side whereas the inverse pattern, with normal ipsilateral R1 and R2 responses with delayed contralateral R2 was found after right stimulation; thus, indicating an incomplete left facial nerve lesion. A laser evoked potential (LEP) examination showed normal responses from both left and right infraorbital region.

\section{Diagnosis}

A diagnosis of "painful tic convulsif due to double vascular impingement" was made as hemifacial spasms occurred in a patient fully satisfying IHS criteria [4] for trigeminal neuralgia.

\section{Therapy}

As the initial CBZ treatment (800 mg/die) was unsuccessful, we started lamotrigine (LTG) with slow titration (adding $25 \mathrm{mg}$ per week) to the final dose of $50 \mathrm{mg}$ b.i.d. LTG led to a satisfactory control of pain with a reduction of episodes from 4-6 per day to 1-2 per week. Considering the good response to drug the neurosurgeon decided to delay surgical microvascular decompression.

\section{Discussion}

"Painful tic convulsif" is a rare disorder first described by Cushing [2] in three patients, characterized by a double
Fig. 1 Parasagittal scan for the study of the Vth nerve

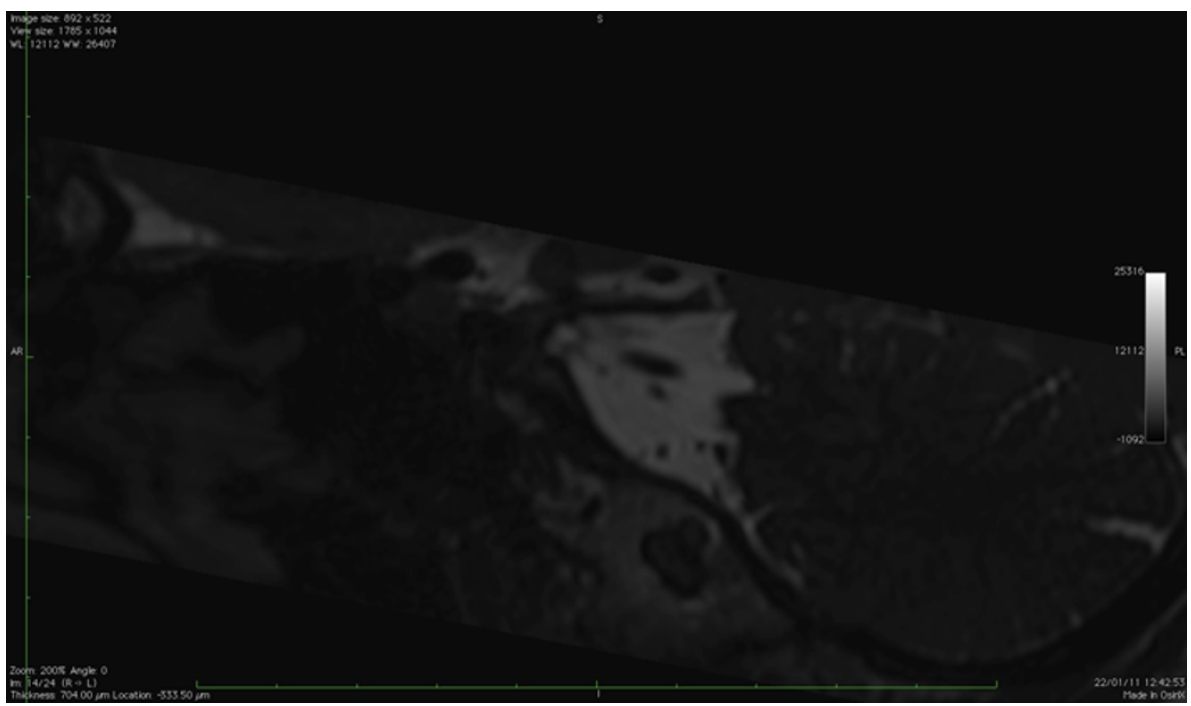


Fig. 2 Coronal scan for the study of the VIIth nerve

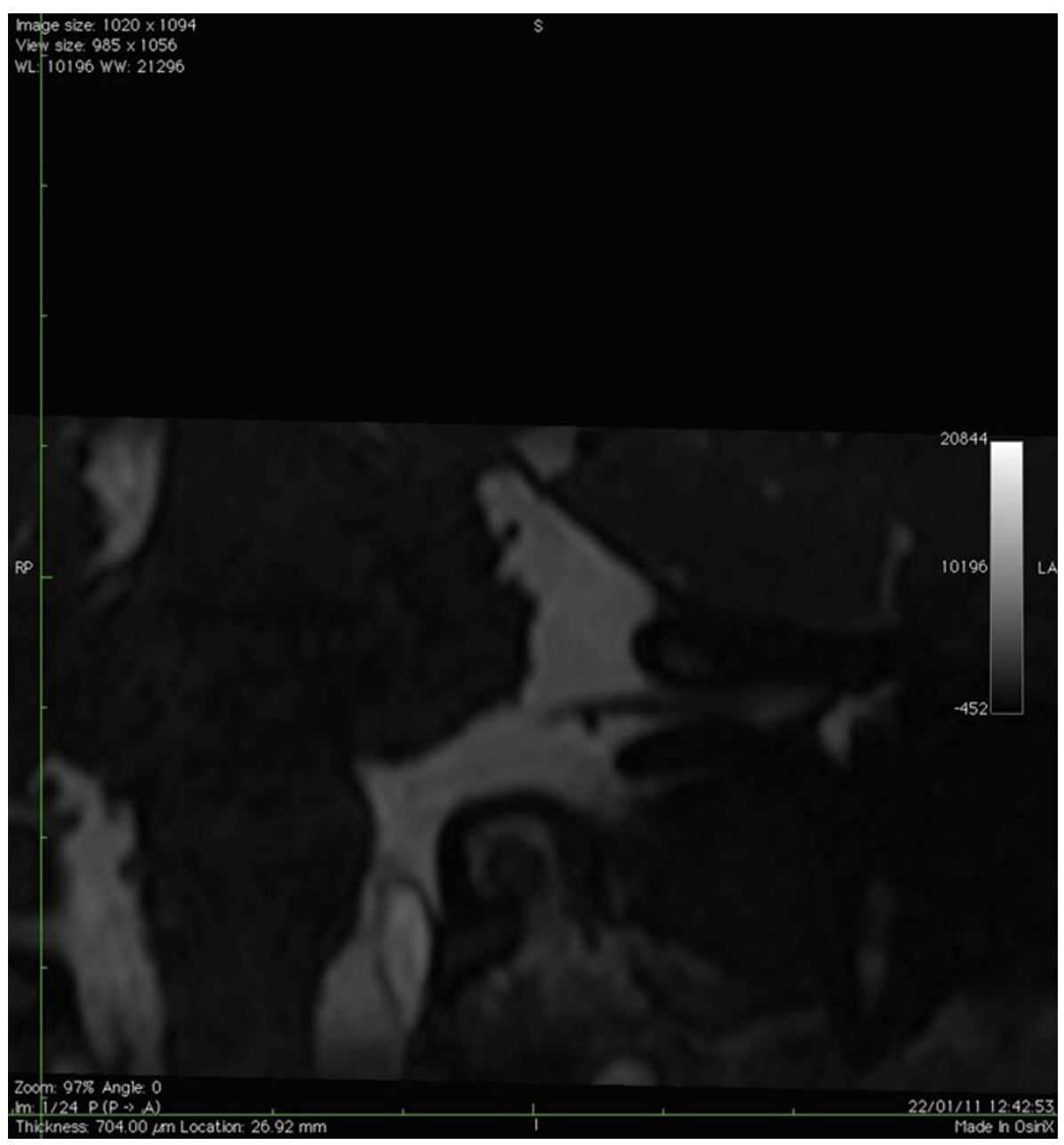

ipsilateral trigeminal and facial irritative syndrome. The commonest cause is vascular compression by ectatic vertebrobasilar artery, even if it may also arise from neoplasms [3, 5]. Pathophysiology of cranial nerve compression rizopathies is still debated as peripheral and central theories are equally considered. Demyelization of axons and subsequent ephactic transmission, along with hyperactivity of neurons in the brainstem nuclei, are probably able to induce spontaneous activation of nerves with sensory (V) and motor (VII) symptoms [3]. In our patient a mild deficit of VII cranial nerve was also clinically and electrophysiologically evident, suggesting that initial damage of neural fibers could also be present. Conversely the trigeminal nerve showed only functional impairment. Most "painful tic convulsif" cases reported in literature presented with involvement of VII preceding the V [5], while in our patient the inverse pattern occurred. This sequence could lead us to consider the facial spasm as due to pain, resembling a simple trigeminal neuralgia, previously called "tic doloreux" by French authors. However, the impairment of the VII cranial nerve, as showed by clinical and neurophysiological findings is not in favour of such interpretation and should be regarded as an independent phenomenon likely due to the neuro-vascular impingement. A meticulous history recording and prolonged observation during physical exam should be performed in all cases of trigeminal neuralgia to avoid underestimation of a possible "painful tic convulsif".

\section{Conflict of interest None.}

Open Access This article is distributed under the terms of the Creative Commons Attribution License which permits any use, distribution and reproduction in any medium, provided the original author(s) and source are credited.

\section{References}

1. Tsuyumu M, Kohmo Y (1991) Painful tic convulsif: case report. Surg Neurol 4:310-313

2. Cushing H (1920) The major trigeminal neuralgias and their surgical treatment based on experiences with 332 gasserian operations. Am J Med Sci 160:157-184

3. Fonoff ET et al (2009) Neurovascular compression in painful tic convulsif. Acta Neurochir 151:989-993 
4. Headache Classification Committee of the International Headache Society (2004) The international classification of headache disorders. Cephalalgia 24(Suppl 1):1-160
5. Zhong et al (2011) Microvascular decompressions in patients with coexistent hemifacial spasm and trigeminal neuralgia. Neurosurgery 68:916-920 